\title{
Effect of ambient extracellular glutamate on Drosophila glutamate receptor trafficking and function
}

\author{
Kaiyun Chen · Hrvoje Augustin · David E. Featherstone
}

Received: 1 August 2008 / Revised: 25 September 2008 / Accepted: 26 September 2008 / Published online: 21 October 2008

(C) The Author(s) 2008. This article is published with open access at Springerlink.com

\begin{abstract}
Measurements suggest that the hemolymph glutamate concentrations in Drosophila are relatively high. This raises the possibility that extracellular glutamate could be an important regulator of glutamatergic transmission in vivo. Using voltage clamp electrophysiology, we found that synaptic currents in D. melanogaster larval neuromuscular junctions are reduced by extracellular glutamate (EC50: $\sim 0.4 \mathrm{mM}$ ), such that only $10-30 \%$ of receptors were functionally available in $1 \mathrm{mM}$ extracellular glutamate. The kinetics of synaptic currents were also slowed in a dosedependent fashion (EC50: $\sim 1 \mathrm{mM}$ ), consistent with the idea that extracellular glutamate preferentially removes the fastest-desensitizing receptors from the functional pool. Prolonged exposure (several hours) to extracellular glutamate also triggers loss of glutamate receptor immunoreactivity from neuromuscular junctions. To determine whether this receptor loss requires that glutamate bind directly to the lost receptors, we examined glutamatedependent loss of receptor immunoreactivity in larvae with glutamate receptor ligand binding mutations. Our results suggest that glutamate-dependent receptor loss requires binding of glutamate directly to the lost receptors. To determine whether lost receptor protein is degraded or merely redistributed, we used immunoblots. Results suggest that glutamate receptor protein is redistributed, but not degraded, after prolonged exposure to high extracellular glutamate.
\end{abstract}

K. Chen and H. Augustin contributed equally to this work.

K. Chen $\cdot$ H. Augustin $\cdot$ D. E. Featherstone $(\square)$

Department of Biological Sciences,

University of Illinois at Chicago,

840 W. Taylor Street (MC 067),

Chicago, IL 60607, USA

e-mail: def@uic.edu
Keywords Drosophila melanogaster - Glutamate · Glutamate receptor $\cdot$ Hemolymph $\cdot$ Synaptic
Abbreviations
EAAT Excitatory amino acid transporter
EJC Excitatory junction current
HRP Horseradish peroxidase
NMJ Neuromuscular junction
EC50 50\% Effective concentration (concentration at which $50 \%$ of effect is observed)

\section{Introduction}

Fruit flies (Drosophila melanogaster) are an increasingly popular model for neurobiological studies due to the numerous genetic tools and techniques available for molecular studies in this organism. Most synaptic examinations in Drosophila target embryonic and/or larval neuromuscular junctions (NMJs), as these synapses are reliably accessible in situ throughout development to a wide variety of powerful techniques, including electron microscopy, immunohistochemistry, voltage clamp electrophysiology, and quantifiable fluorescent optical imaging. Drosophila NMJs (as in other arthropods) are also glutamatergic (Jan and Jan 1976). This attribute makes them somewhat comparable to mammalian central synapses, which are subjects of great interest to funding agencies.

The fact that Drosophila NMJs are glutamatergic also raises a mystery, because arthropod hemolymph in general, and Drosophila hemolymph in particular, has long been known to contain a relatively high concentration of glutamate (McDonald 1975; Echalier 1997; Pierce et al. 1999; Augustin et al. 2007; Piyankarage et al. 2008). Depending on the study and sampling conditions, measurements of 
hemolymph glutamate in $D$. melanogaster typically range from 0.5 to $10 \mathrm{mM}$ or more (Echalier 1997), with the average being 1-2 $\mathrm{mM}$ (Augustin et al. 2007). It is not clear what the source of measurement variation is between studies. As all cells tend to sequester and metabolize glutamate, some measurement variation could be due to differences in hemolymph extraction, or contamination by hemocytes (Ribeiro and Brehelin 2006). Variation between studies could also be due to circadian or behaviorally-associated changes in hemolymph composition. For example: longterm dialysis of unanesthetized freely-moving crayfish shows dramatic (twofold) changes in hemolymph glutamate associated with the onset of nighttime (Cebada et al. 2006), and our own preliminary observations (S.C. Piyankarage et al., unpublished) suggest that glutamate levels in Drosophila larval hemolymph also change two to threefold depending on behavioral state.

The mechanisms controlling hemolymph glutamate are not clear. The Drosophila genome encodes two members of the excitatory amino acid transporter (EAAT) family, which remove glutamate from the extracellular space, but only one of these genes appears to encode a high-affinity glutamate transporter (the other shows preference for aspartate) (Seal et al. 1998; Besson et al. 1999, 2000). Surprisingly, Drosophila EAAT transporters do not appear to be localized near embryonic or larval NMJs (Rival et al. 2006), suggesting that glutamatergic transmission in these synapses is normally terminated by diffusion of glutamate and subsequent dilution by hemolymph. Hemolymph measurements in Drosophila EAAT mutants have not been performed, so it is unclear how much Drosophila EAAT activity regulates hemolymph glutamate.

The Drosophila genome also encodes five members of the $\mathrm{xCT}$ protein family. $\mathrm{xCT}$ proteins, in combination with $4 \mathrm{~F} 2 \mathrm{hc}$ proteins, form cystine-glutamate transporters that export glutamate in exchange for cystine (Augustin et al. 2007). Drosophila larvae with mutations in one of these $\mathrm{xCT}$ proteins, called 'genderblind' based on a mutant courtship phenotype, have one-half normal hemolymph glutamate (Augustin et al. 2007; Piyankarage et al. 2008). Thus, Drosophila hemolymph glutamate appears heavily regulated by cystine-glutamate transport. It is not known whether $\mathrm{xCT}$ activity might be regulated by photoperiod or behavioral state.

Regardless of the mechanisms controlling hemolymph composition, how do Drosophila NMJs perfused by hemolymph maintain glutamatergic transmission in the presence of high glutamate concentrations? D. melanogaster NMJ glutamate receptors have a relatively high EC50 for activation—about $2 \mathrm{mM}$ (Heckmann et al. 1996)—but the EC50 for glutamate receptor desensitization can be substantially lower (Featherstone and Shippy 2008). Thus, it would seem that glutamatergic transmission in Drosophila NMJs might be prohibited by receptor desensitization. But obviously it is not.

Researchers have proposed various mechanisms by which hemolymph glutamate might be excluded from arthropod NMJs. For example, hemolymph might be physically excluded from NMJs by ensheathing glia. Indeed, glia that might serve this purpose have been observed in Drosophila larvae (Banerjee et al. 2006; Augustin et al. 2007). Hemolymph glutamate could also be rendered physiologically inert by being bound to metal ions or certain proteins (Faeder and Salpeter 1970; Evans 1972; Clements and May 1974; Evans and Crossley 1974), though such glutamate would still have to be easily isolated to be measured by analytical chemistry.

But high ambient extracellular glutamate and glutamatergic transmission need not be mutually exclusive. Indeed, recent work in D. melanogaster suggests that extracellular glutamate might serve an important modulatory role (Augustin et al. 2007; Grosjean et al. 2008). In this model, hemolymph glutamate constitutively desensitizes a subset of glutamate receptors to regulate glutamatergic synapse strength. The precise degree to which this might happen, however, has not previously been determined. Here, we provide a detailed voltage-clamp analysis of the effects of ambient extracellular glutamate on Drosophila neuromuscular transmission, and examine mechanisms by which prolonged exposure to extracellular glutamate causes loss of postsynaptic glutamate receptor protein.

\section{Materials and methods}

All experiments were performed on wandering third instar larvae of D. melanogaster. Except where noted, all data were obtained from wild-type strain 'Oregon $R$ ' or (where appropriate) the physiologically equivalent control strain w[1118]. In GluRIIA[E783A]; df(2L)cl[h4]/GluRIIA[AD9] mutant animals, all GluRIIA proteins are engineered such that there is an alanine in place of glutamic acid at position 783. This mutation (E783A) eliminates glutamate binding, and has been previously described (Schmid et al. 2006).

Two-electrode voltage clamp electrophysiology was performed as previously described (Liebl et al. 2005; Augustin et al. 2007). Briefly, ventral longitudinal muscle 6 in abdominal segments 3-4 of wandering third instar larvae was voltage-clamped at $-60 \mathrm{mV}$ using an Axon Instruments/MDS Analytical Technologies (Sunnyvale, CA, USA) GeneClamp 500B amplifier, under standard Drosophila saline $(135 \mathrm{mM} \mathrm{NaCl}, 5 \mathrm{mM} \mathrm{KCl}, 4 \mathrm{mM}$ $\mathrm{MgCl}_{2}, 1.8 \mathrm{mM} \mathrm{CaCl}_{2}, 5 \mathrm{mM}$ TES, $72 \mathrm{mM}$ sucrose), with various concentrations of L-glutamate added to the bath as indicated. Intracellular electrodes contained $3 \mathrm{M} \mathrm{KCl}$. To record excitatory junction currents (EJCs), segmental 
nerves to the voltage-clamped muscle were stimulated at $0.5 \mathrm{~Hz}$ by a Grass S48 stimulator via a suction electrode filled with bath saline ( $0 \mathrm{mM}$ glutamate). Electrodes were fashioned from thin-walled borosilicate glass capillaries with filaments (TW100F-4; World Precision Instruments, Sarasota, FL, USA) using a Sutter Instrument (Novato, CA, USA) P-2000 glass pipette puller. Typical electrode resistance was 10-20 MOhms. All electrophysiological recordings occurred within minutes of dissection in experimental saline, and were digitized by a Digidata 1322A digitizer (Axon Instruments). PClamp 10 software (Axon Instruments) was used for data acquisition and analysis. Time constants were calculated from exponential fits to the falling phase of individual currents, using PClamp 10.

Immunohistochemistry and confocal microscopy were performed as previously described (Featherstone et al. 2002, 2005; Chen et al. 2005; Liebl et al. 2005; Augustin et al. 2007). All data are from NMJs on ventral longitudinal muscles 6 and 7 in abdominal segments 3-4 of wandering third instar larvae. Culture of semi-intact larval NMJ preps was performed as previously described (Ball et al. 2003; Augustin et al. 2007). Briefly, filleted third instar larvae were incubated at room temperature $\left(\sim 22^{\circ} \mathrm{C}\right)$ in minimal saline supplemented with $0.1 \mathrm{mg} / \mathrm{ml}$ gentamycin, $2 \%$ heatinactivated fetal calf serum, and (when indicated) L-glutamate. The incubation medium was changed thrice daily. For the experiments described here, preparations were incubated $24 \mathrm{~h}$ before fixation for immunohistochemistry or protein isolation for immunoblots.

Immunohistochemical measurements of postsynaptic glutamate receptor abundance were made by using ImageJ software to quantify mean postsynaptic immunofluorescence intensity relative to fluorescence in surrounding muscle tissue $\left(F_{\text {synapse }} / F_{\text {background membrane }}\right)$ Postsynaptic immunofluorescence was delimited by horseradish peroxidase (HRP) staining in the second confocal channel, as previously described (Augustin et al. 2007). Essentially, we outlined the NMJ manually using image analysis software, then measured total glutamate receptor immunofluoresence in this area using the software's tools. We then moved the same outline for measurement to a nearby region on the same muscle and measured fluorescence in the same way for the same channel. The first measurement, which represents postsynaptic glutamate receptor immunoreactivity, was then divided by the second number, which represents background muscle fluorescence.

Immunoblots were performed as previously described (Liebl et al. 2005, 2006). Before loading on the gel, total protein was quantified with a Bradford assay, and a precise quantity (indicated in figure) loaded into each lane. This allowed us to control for protein extraction and loading, as well as ensure that blots had sensitivity to detect changes in glutamate receptor protein. Immunoreactivity was detected using chemiluminescence imaged with a Bio-Rad Versadoc 4000 and quantified using Quantity One software (Bio-Rad Laboratories, Hercules, CA, USA). Note that the antibody used for visualization of GluRIIB in situ (Fig. 3) differs from that used for immunoblots (Fig. 4). The antibody used to visualize GluRIIB in NMJs is based on that originally described by Marrus et al. (2004), but we have never achieved good results with this antibody for immunoblots. For immunoblots, we use a GluRIIB antibody raised against a slightly longer epitope, as previously described (Liebl et al. 2005). Although specific for GluRIIB on immunoblots, this latter GluRIIB antibody does not work in situ.

Mouse monoclonal anti-GluRIIA (8B4D2) antibodies were obtained from University of Iowa Developmental Studies Hybridoma bank and used at 1/100. Rabbit polyclonal anti-GluRIIB antibodies for in situ work were generated by the UIC antibody facility based on a previously described peptide epitope (Marrus et al. 2004), and used at 1/1000. Rabbit polyclonal anti-GluRIIB antibodies for immunoblots were generated by SynPep (Dublin, CA, USA), and used at 1/400, as previously described (Liebl et al. 2005). FITC, TRITC, and Cy5-cojugated goat antimouse or anti-rabbit secondary antibodies were obtained from Jackson Immunoresearch Laboratories (West Grove, PA, USA) and used at 1/400. TRITC- or Cy5-conjugated anti-HRP antibodies were also obtained from Jackson Immunoresearch, and used at 1/100.

Unless otherwise noted, statistics are mean \pm standard error, and $n=$ number of animals. In figures, $* * *$ represents $P<0.001 ;$ N.S. indicates not significant $(P>0.05)$.

\section{Results}

Figure 1a shows EJCs recorded from third instar larval muscle 6 in various concentrations of ambient extracellular glutamate. In $0 \mathrm{mM}$ extracellular glutamate, stimulation of the segmental nerve elicited robust EJCs. These EJCs were over $200 \mathrm{nA}$ in amplitude and lasted less than $50 \mathrm{~ms}$ (Fig. 1). As extracellular glutamate concentration was raised, EJC amplitudes decreased and EJC falling phase kinetics slowed (Fig. 1). In $0.5 \mathrm{mM}$ glutamate, EJC amplitudes were reduced to less than $50 \mathrm{nA}$ and kinetics were slowed such that EJCs lasted 100-200 ms (Fig. 1). Above $1 \mathrm{mM}$ glutamate, EJC amplitudes and kinetics were further altered in a dose-dependent fashion (Fig. 1a; note scale bar change between first and second row of examples). EJCs were still detectable in $2.5 \mathrm{mM}$ extracellular glutamate, but were very small (<10 $\mathrm{nA})$ and slow (>300 ms). In $3 \mathrm{mM}$ extracellular glutamate, EJCs were undetectable.

As shown in Fig. 1b, the dose-response relationship between EJC amplitude and extracellular glutamate is 


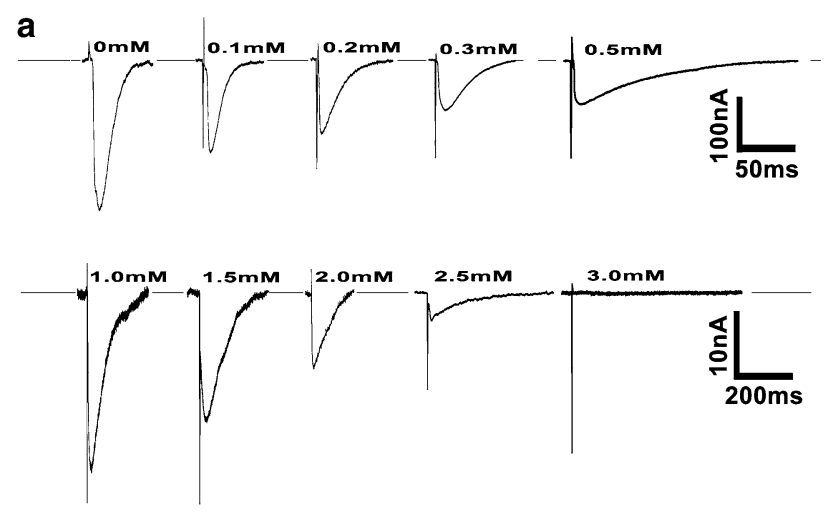

b
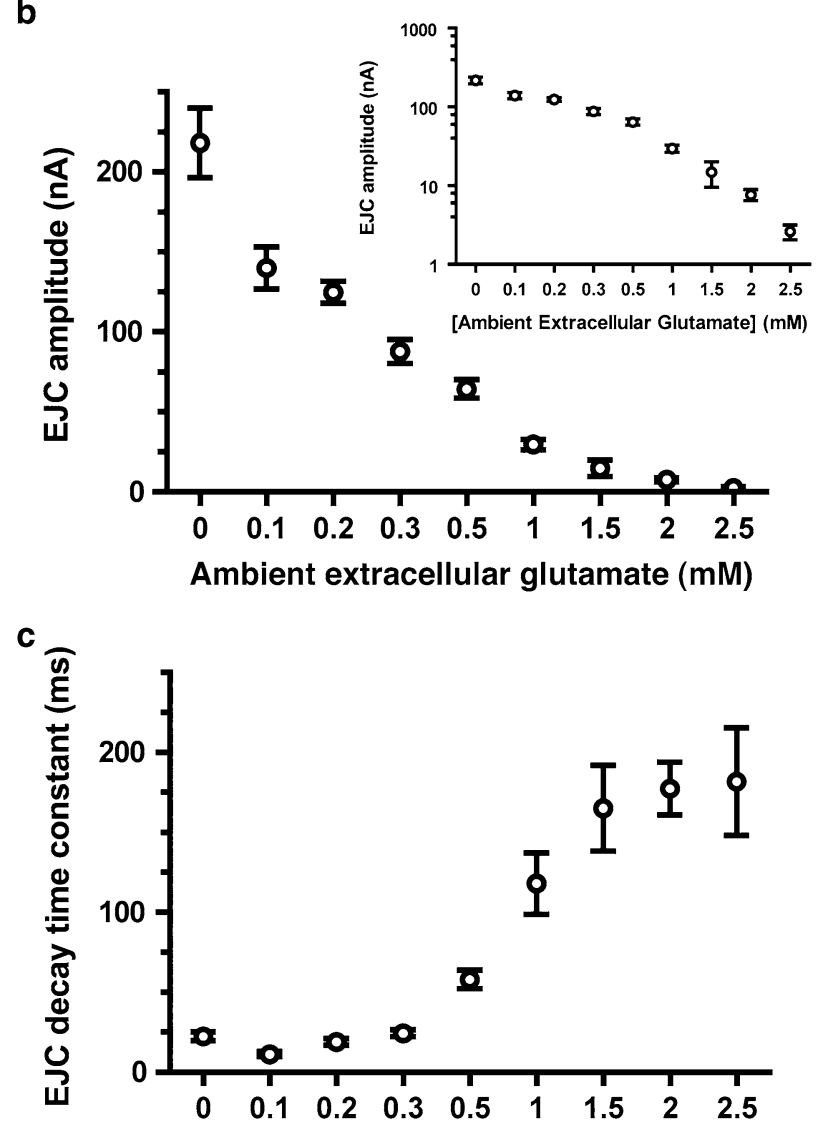

Ambient extracellular glutamate (mM)

Fig. 1 Excitatory junction current (EJC) amplitude is decreased, and duration increased, in higher glutamate concentrations. a EJCs recorded in various concentrations of extracellular (bath) glutamate. b EJC amplitude plotted against ambient extracellular (bath) glutamate concentration. Inset The same data plotted on a log scale. c EJC decay time constant plotted against ambient extracellular (bath) glutamate concentration

relatively broad, with no clear threshold below which ambient extracellular glutamate has no effect. Presumably, therefore, almost any concentration of ambient extracellular glutamate in vivo reduces neuromuscular transmission. Postsynaptic muscle contraction, however, depends on several factors including summation of postsynaptic potentials and postsynaptic calcium influx. Postsynaptic summation and calcium influx correlate primarily with EJC integral rather than EJC amplitude. EJC kinetics (and thus EJC integral) are expected to change with increased extracellular glutamate because the first receptors to functionally disappear with increasing extracellular glutamate are presumably those that desensitize most readily. Therefore, we also measured the effect of ambient extracellular glutamate on EJC kinetics.

As shown in Fig. 1c, EJC decay time constants in low ambient extracellular glutamate were relatively fast ( $<20 \mathrm{~ms}$ ). However, these time constants increased rapidly above $0.3 \mathrm{mM}$ extracellular glutamate, to a maximum of approximately $175 \mathrm{~ms}$ at $1.5-2 \mathrm{mM}$ glutamate (Fig. 1c). Thus, as expected, the most readily desensitized glutamate receptors were preferentially eliminated from the functional pool by glutamate, leading to a glutamate-dependent change in EJC kinetics. This large glutamate-dependent increase in EJC time constants might physiologically offset, to some extent, the consequences of reduced EJC amplitude in vivo.

We also examined the effects of ambient extracellular glutamate on spontaneous miniature excitatory junction currents (sEJCs). As shown in Fig. 2a, voltage-clamp recordings in $0 \mathrm{mM}$ extracellular glutamate showed abundant sEJCs, at an average frequency of $3.1 \pm 0.3 \mathrm{~Hz}$ $(n=4)$. As extracellular glutamate concentration increased, sEJC frequency decreased $(0.1 \mathrm{mM}=2.2 \pm 0.4 \mathrm{~Hz}, n=6$; $0.2 \mathrm{mM}=1.7 \pm 0.1 \mathrm{~Hz}, \quad n=4 ; \quad 0.3 \mathrm{mM}=1.7 \pm 0.4 \mathrm{~Hz}$, $n=4 ; 0.5 \mathrm{mM}=1.3 \pm 0.4 \mathrm{~Hz}, n=4$ ) (Fig. 2c). sEJCs were not reliably detectable at $1.0 \mathrm{mM}$ extracellular glutamate or higher concentrations.

The sEJC amplitude also decreased as extracellular glutamate concentration increased (Fig. 2b, d). For example, the average sEJC amplitude in $0 \mathrm{mM}$ extracellular glutamate was $0.83 \pm 0.07 \mathrm{nA}(n=4)$, but only $0.32 \pm 0.02 \mathrm{nA}$ $(n=4)$ in $0.5 \mathrm{mM}$ extracellular glutamate (Fig. 2d).

Reduced sEJCs frequencies associated with higher extracellular glutamate could be due to altered presynaptic neurotransmitter release or reduced ability to detect increasingly small sEJCs amidst the recording noise. In support of the latter possibility, sEJC amplitude distribution showed marked flattening on the left side near $0.15 \mathrm{nA}$ (Fig. 2b), which was essentially the detectability limit given the noise in our recordings.

The sEJC decay time, like EJC decay time, increased with increasing extracellular glutamate (Fig. 2e). Single channel desensitization time constants of Drosophila larval muscle glutamate receptors have previously been shown to decrease with increasing extracellular glutamate (up to $1 \mathrm{mM}$, after which they increase) (Heckmann and Dudel 1997). Therefore, the increased sEJC decay times we measured also support the idea that increasing extracellular 
a
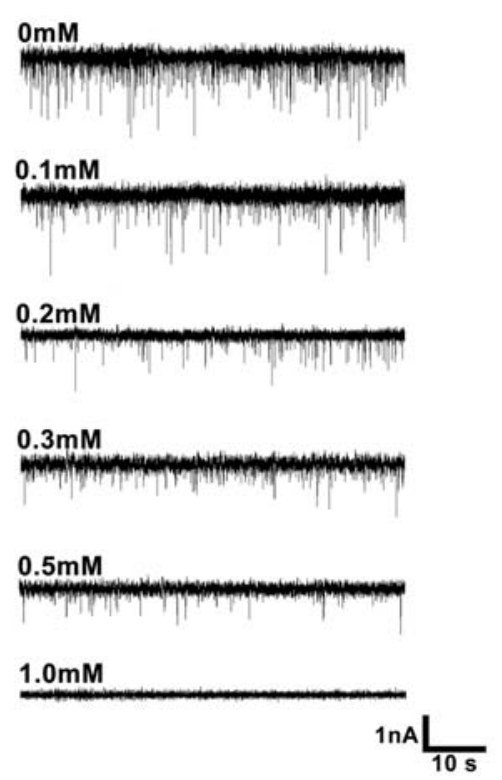

b

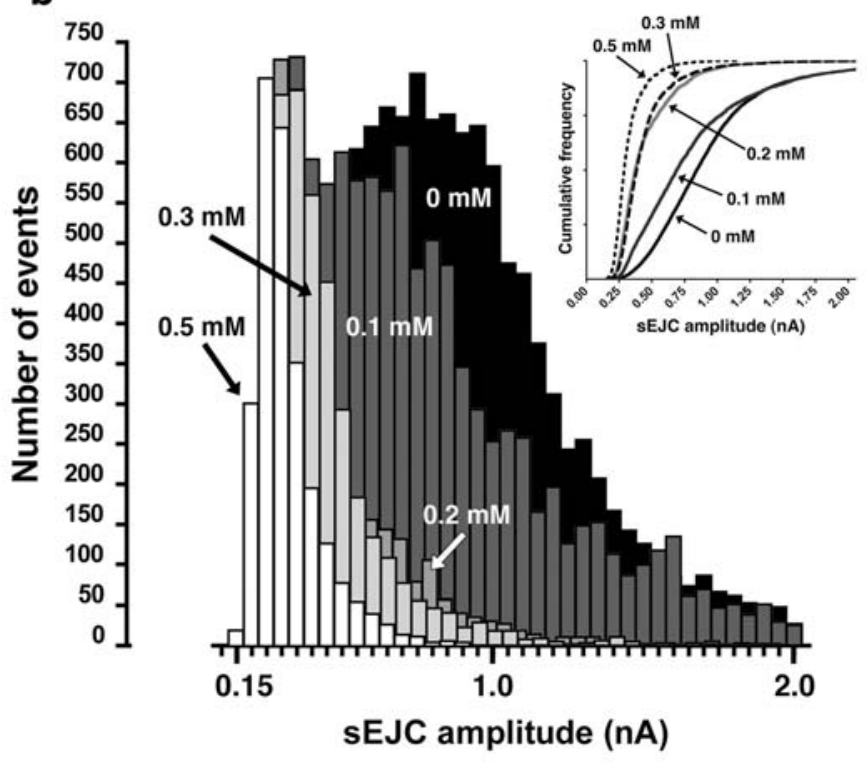

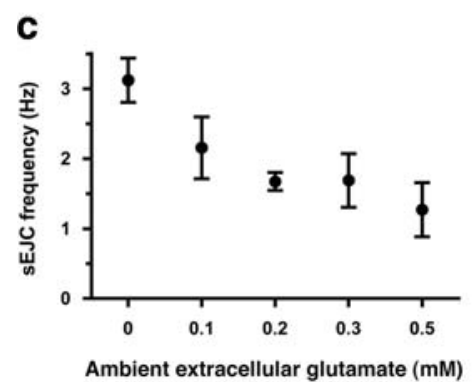

d

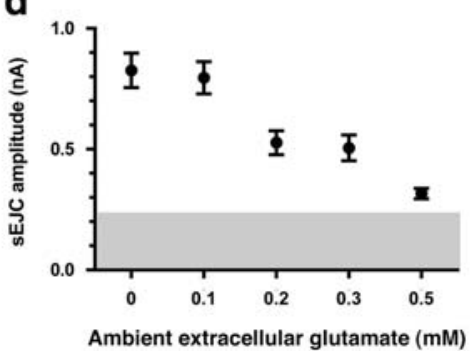

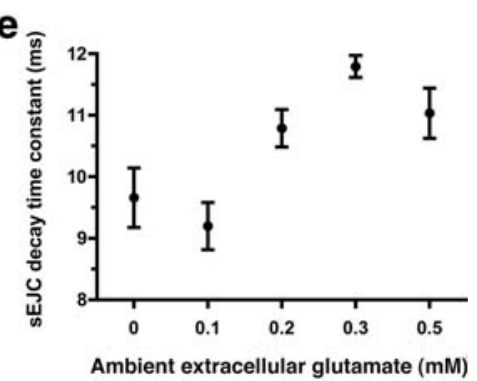

Fig. 2 Spontaneous excitatory junction current (sEJC/'mini') amplitude is decreased, and duration increased, in higher glutamate concentrations. a Continuous voltage clamp recordings recorded at high resolution in various concentrations of extracellular (bath) glutamate. Small downward deflections are sEJCs. b sEJC amplitude histograms from recordings in various concentrations of ambient extracellular (bath) glutamate. Inset Cumulative frequency plot of the same data.

glutamate preferentially desensitizes (and thus removes from the functional population) individual receptors with fastest desensitization.

The data above demonstrate that extracellular glutamate functionally eliminates glutamate receptors within a few minutes. We have previously demonstrated that prolonged exposure to extracellular glutamate also reduces glutamate receptor immunoreactivity in Drosophila larval NMJs over 6-12 h (Augustin et al. 2007). This glutamate-dependent loss of immunoreactivity is desensitization-dependent (Augustin et al. 2007), consistent with the idea that constitutive desensitization leads to redistribution or degradation of postsynaptic glutamate receptors. However, our data do not conclusively determine whether postsynaptic receptor loss requires binding of glutamate directly to the redistributed receptors. It could be the case, for example, that extracellular glutamate activates postsynaptic metabotro- c sEJC frequency plotted against ambient extracellular (bath) glutamate concentration. d sEJC amplitude plotted against ambient extracellular (bath) glutamate concentration. Gray area represents zone where sEJC amplitude would be too small for the sEJC to be reliably distinguished from baseline noise. e sEJC decay time constant plotted against ambient extracellular (bath) glutamate concentration

pic glutamate receptors, and this in turn triggers redistribution or degradation of ionotropic glutamate receptors within the postsynaptic muscle. Indeed, metabotropic glutamate receptors are present in Drosophila larval NMJs (Bogdanik et al. 2004).

To test whether glutamate-dependent loss of postsynaptic glutamate receptors depends on glutamate binding directly to the receptors themselves, we analyzed receptor loss in larvae of the genotype GluRIIA[E783A]; $d f(2 L) c l[h 4] / G l u R I I A[A D 9]$. There are two subtypes of postsynaptic glutamate receptors in Drosophila larval NMJs: A-type receptors and B-type receptors. A-type glutamate receptors are tetramers composed of GluRIIA, GluRIIC (also known as GluRIII), GluRIID, and GluRIIE. B-type glutamate receptors are tetramers composed of GluRIIB, GluRIIC, GluRIID, and GluRIIE (Chen et al. 2005; Featherstone et al. 2005; Qin et al. 2005). In 
Fig. 3 Glutamate-dependent loss of GluRIIA immunoreactivity is blocked in GluRIIA[E783A] mutants. a Synaptic GluRIIA abundance, measured immunohistochemically, after $24 \mathrm{~h}$ incubation in 0 or $2 \mathrm{mM}$ ambient extracellular glutamate, and in wildtype (WT) or GluRIIA[E783A]; $d f(2 L) c l[h 4] / G l u R I I A[A D 9]$ mutant larvae (designated in figure as 'E783A'). b Synaptic GluRIIB abundance, measured immunohistochemically, after $24 \mathrm{~h}$ incubation in 0 or $2 \mathrm{mM}$ ambient extracellular glutamate, and in WT or GluRIIA[E783A]; $d f(2 L) c l[h 4] / G l u R I I A[A D 9]$ mutant larvae (designated in figure as 'E783A'). c Confocal micrographs of muscle $6 / 7$ NMJs stained with anti-GluRIIA (left three rows of panels) or anti-GluRIIB (right two rows of panels), and anti-HRP (which stains the presynaptic terminal)
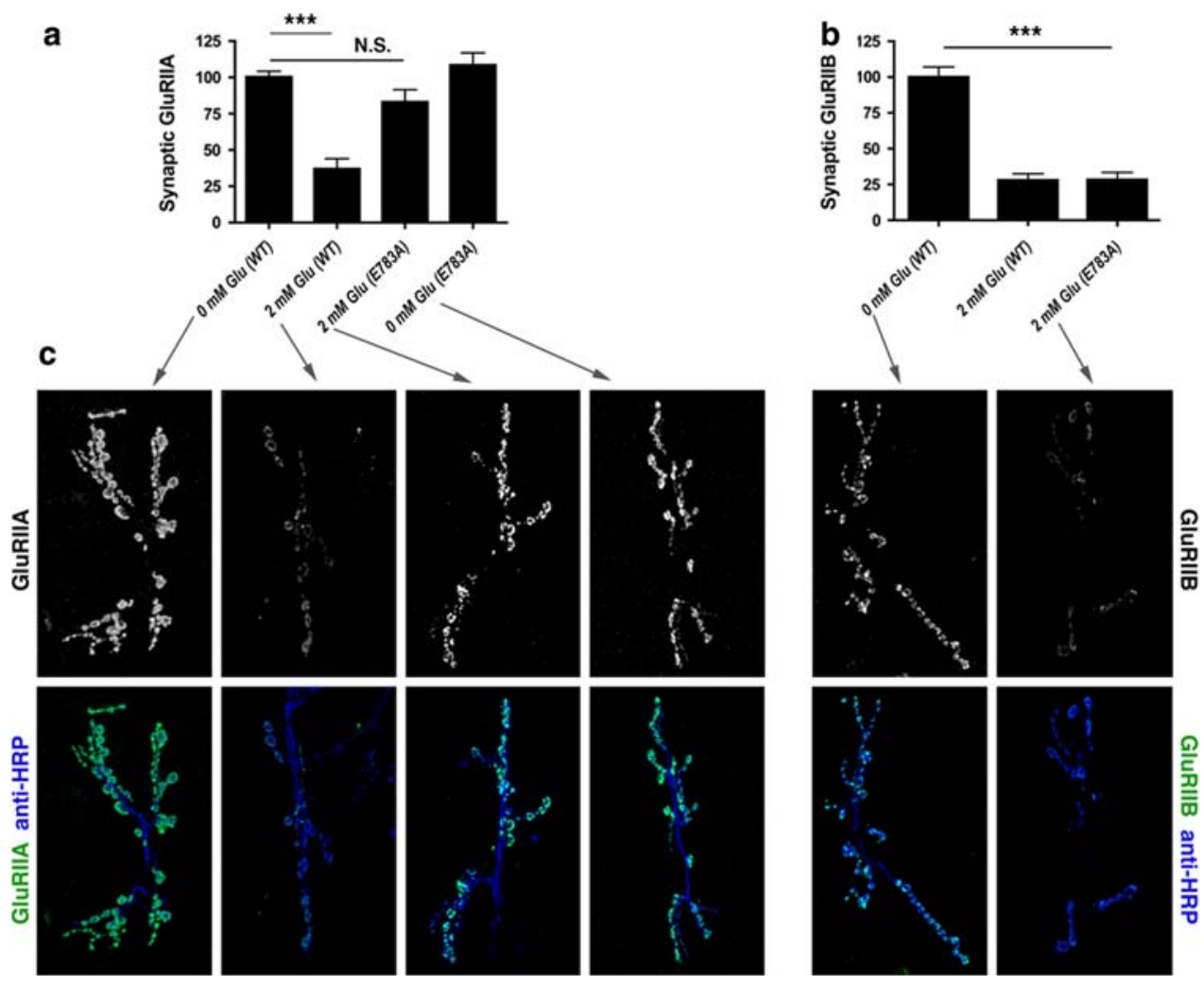

GluRIIA[E783A]; df(2L)cl[h4]/GluRIIA[AD9] larvae, all A-type receptors contain GluRIIA subunits with the mutation E783A, which disrupts glutamate binding. Thus, A-type receptors in this genotype cannot be activated or desensitized. B-type receptors in the same synapse, however, are wildtype. If glutamate-dependent loss of postsynaptic receptors requires that glutamate bind directly to the receptors that are lost, then A-type receptors-and only A-type receptors-in these mutant larvae will be 'immune' to glutamate-dependent receptor loss.

As shown in Fig. 3 and in our previous work (Augustin et al. 2007), application of $2 \mathrm{mM}$ extracellular glutamate to semi-intact wildtype larvae for $24 \mathrm{~h}$ reduces GluRIIA and GluRIIB immunoreactivity to approximately one-third normal. In GluRIIA[E783A]; df(2L)cl[h4]/GluRIIA[AD9] larvae, however, loss of GluRIIA immunoreactivity was blocked. This block is not an artifact of basally increased A-type receptors in GluRIIA[E783A]; df(2L)cl[h4]/GluRIIA[AD9] larvae, because we observed no increase in GluRIIA immunoreactivity in mutant larvae exposed to $0 \mathrm{mM}$ glutamate (Fig. 3a, c). The block was also specific to A-type receptors, because glutamate-dependent loss of GluRIIB immunoreactivity was not prevented (Fig. 3b, d). Based on these data, we conclude that glutamate must bind to receptors directly to induce their loss from the NMJ.

Where does the glutamate receptor protein go? Loss of NMJ immunoreactivity could be caused by either redistribution or degradation of postsynaptic glutamate receptor protein. As a first step in answering this question, we used immunoblots to determine whether extracellular glutamate causes degradation of glutamate receptor protein.

As shown in Fig. 4, GluRIIA and GluRIIB protein from larvae were detectable on immunoblots. According to these immunoblots, exposure to $2 \mathrm{mM}$ glutamate for $24 \mathrm{~h}$ causes no significant reduction in total GluRIIA or GluRIIB protein, despite the fact that these same conditions reduce GluRIIA and GluRIIB immunoreactivity in NMJs to approximately one-third normal (Fig. 3). Our immunoblot technique was sensitive enough to detect a one-half reduction in GluRIIA or GluRIIB protein, since loading reduced amounts of protein showed easily measurable concomitant reductions in GluRIIA and GluRIIB (Fig. 4). Our detection was also specific to GluRIIA and GluRIIB, because protein isolated from GluRIIA[SP22] mutant animals, in which the GluRIIA and GluRIIB genes are deleted, showed no bands of appropriate size (Fig. 4). It is unlikely that GluRIIA and GluRIIB from other (non NMJ) tissues overwhelmed our ability to detect a decrease in NMJ receptor protein, since GluRIIA and GluRIIB appear to be expressed only in body wall muscles (Petersen et al. 1997; Qin et al. 2005). We therefore conclude that prolonged exposure to extracellular glutamate redistributes and disperses postsynaptic A-type and B-type glutamate receptors sufficient to make them immunohistochemically invisible, but does not trigger protein degradation. It should be noted that our methods cannot determine whether glutamate causes GluRIIA and 


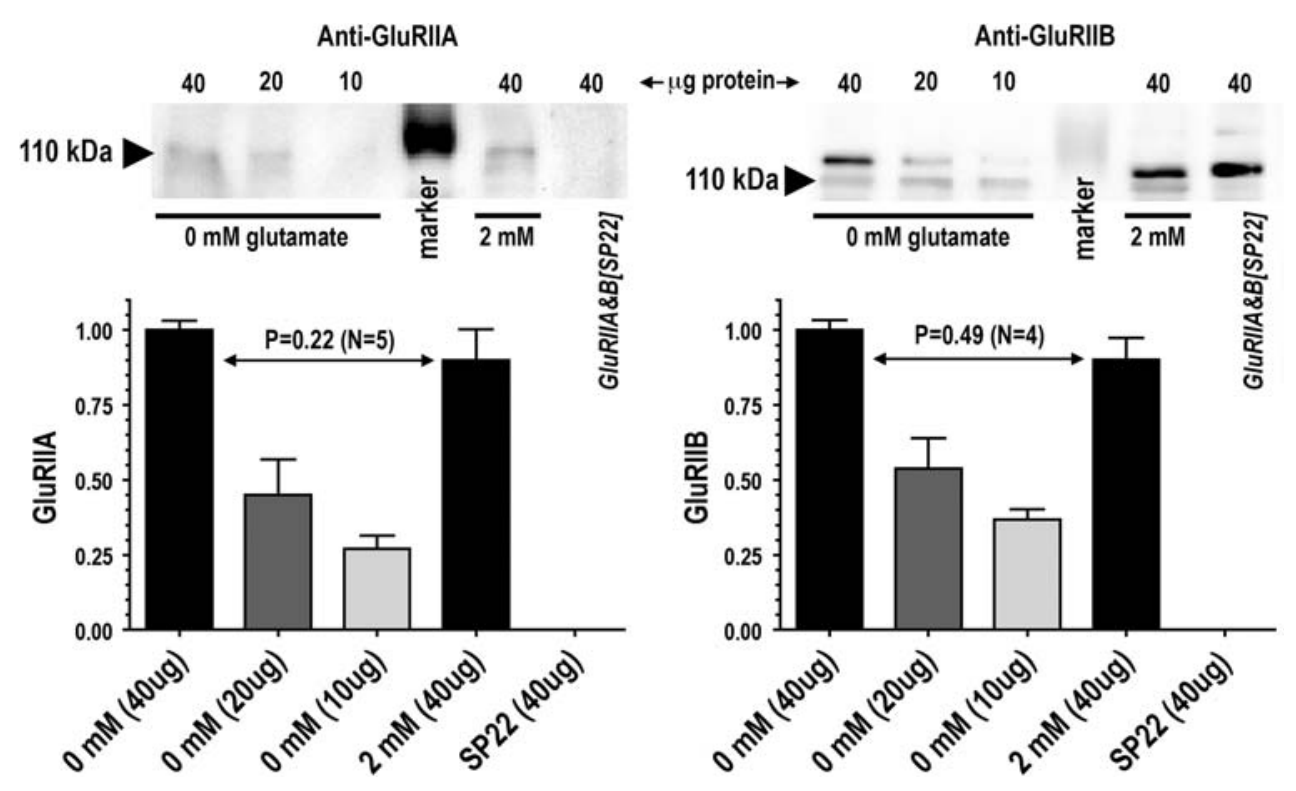

Fig. 4 Twenty-four hours incubation in $2 \mathrm{mM}$ glutamate does not reduce GluRIIA or GluRIIB protein levels. Top Portions of immunoblots showing anti-GluRIIA immunoreactivity (left blot) or anti-GluRIIB immunoreactivity (right blot). For each blot, lanes 1, 5, and 6 were loaded with $40 \mu \mathrm{g}$ of protein; lane 2 was loaded with $20 \mu \mathrm{g}$ of protein, and lane 3 was loaded with $10 \mu \mathrm{g}$ of protein. Lane 4 was used

for protein size markers (not fully visible). Lanes $1-3$ were loaded with protein isolated from larvae cultured $24 \mathrm{~h}$ in $0 \mathrm{mM}$ glutamate. Lane 5 was loaded with protein isolated from larvae cultured $24 \mathrm{~h}$ in $2 \mathrm{mM}$ glutamate. Lane 6 was loaded with protein isolated from $D f(2 L) S P 22$ (AKA GluRIIA\&B[SP22]) embryos, in which the GluRIIA and GluRIIB genes are deleted

GluRIIB to be dispersed in the muscle cell membrane or internalized. Our methods also cannot determine whether redistributed GluRIIA and GluRIIB protein remains assembled with GluRIIC, GluRIID, and GluRIIE. These are important questions for the future, since they determine the speed by which receptors could be re-recruited to the postsynaptic density following a decrease in ambient extracellular glutamate.

\section{Discussion}

Our results demonstrate that synaptic transmission in Drosophila larval NMJs is reduced in a dose-dependent manner by ambient extracellular glutamate (EC50: $\sim 0.4 \mathrm{mM}$ ). The kinetics of synaptic currents are also slowed by increasing extracellular glutamate (EC50: $\sim 1 \mathrm{mM}$ ), consistent with the idea that ambient extracellular glutamate preferentially removes the fastest-desensitizing receptors from the functional pool, and raising the possibility that increased summation of endplate potentials and postsynaptic muscle calcium influx in vivo may temper the effects of reduced synaptic transmission. As previously reported, prolonged exposure to ambient extracellular glutamate causes loss of glutamate receptor immunoreactivity from NMJs (EC50: 1-2 mM). To determine whether the glutamate-dependent loss of postsynaptic receptors involves glutamate binding directly to the lost receptors, we

examined glutamate-dependent receptor loss in larvae carrying GluRIIA[E783A] mutations that block glutamatebinding in a subset of neuromuscular receptors. Glutamate receptors that could not bind glutamate were not lost after prolonged exposure to glutamate, suggesting that activation and/or desensitization is a prerequisite for loss of the same postsynaptic glutamate receptors. This is consistent with our previous work, which showed that desensitization inhibitors also block glutamate-dependent loss of postsynaptic glutamate receptor immunoreactivity (Augustin et al. 2007). To determine whether lost receptors are degraded or merely redistributed, we measured total GluRIIA and GluRIIB protein using immunoblots. Only GluRIIA and GluRIIB were examined because other NMJ subunits (GluRIIC, GluRIID, and GluRIIE) are not expressed exclusively in larval body wall muscle. Our immunoblot experiments suggested that extracellular glutamate triggers glutamate receptor protein redistribution, rather than degradation.

Taken together, the data here and in previous publications (Heckmann et al. 1996; Heckmann and Dudel 1997; Augustin et al. 2007) suggest that, when exposed to glutamate, Drosophila NMJ glutamate receptors are activated (within microseconds), then desensitized (within milliseconds), and eventually redistributed (within hours). Interestingly, the EC50s for glutamate-triggered activation $(\sim 2 \mathrm{mM})$, desensitization $(\sim 0.4 \mathrm{mM})$, and redistribution $(\sim 1-2 \mathrm{mM})$ differ. This suggests that glutamate receptor channel desensitization can be triggered in closed states by 
glutamate binding to any or all four of the individual glutamate receptor subunits (which may have slightly different glutamate binding affinities and/or display cooperativity), while activation and redistribution requires that glutamate bind to all four subunits. The fact that redistribution of A-type receptors was blocked in GluRIIA[E783A] mutants even though only one subunit in each receptor could not bind glutamate is consistent with this model.

The mechanism by which prolonged exposure to glutamate causes loss of postsynaptic glutamate receptors is unknown. Glutamate could cause postsynaptic receptors to be removed from the synapse, or could alternatively stabilize receptors as they transit through extrasynaptic pools, such that they are unable to accumulate in the NMJ. The latter hypothesis is consistent with time-course data showing that ambient extracellular glutamate prevents normal slow accumulation of receptors in NMJs, and does not cause net loss of receptors from the NMJ (Augustin et al. 2007). Elegant live imaging of A- and B-type receptor cluster formation in Drosophila larval NMJs suggests that presynaptic glutamate release suppresses postsynaptic clustering of A-type receptors, but simultaneously promotes postsynaptic incorporation of B-type receptors (Schmid et al. 2008). At first thought, this appears to be inconsistent with our findings showing that glutamate causes loss of both A- and B-type receptors from NMJs. But ambient extracellular glutamate, in contrast to glutamate released from presynaptic vesicles, is not locally distributed. If postsynaptic A-type receptors are destabilized and dispersed from NMJs by glutamate, while extrasynaptic B-type receptors are stabilized, then ambient extracellular glutamate may simultaneously disperse postsynaptic A-type receptors and stabilize extrasynaptic B-type receptors. In this way, live imaging (Schmid et al. 2008) and our immunohistochemical studies (Augustin et al. 2007) are reconciled.

In summary, our results suggest that, depending on hemolymph glutamate concentration in vivo, only a subset of slowly desensitizing glutamate receptors may be functionally available in the D. melanogaster NMJ. The rest of the receptors are held in reserve pools of desensitized and/ or redistributed receptors. This raises the possibility that regulation of hemolymph glutamate could be an important mechanism for synaptic modulation in Drosophila. Unfortunately, little is known regarding changes in hemolymph glutamate or how such changes might be regulated. These are therefore potentially important topics for future study.

Acknowledgments We would like to thank Dr Stephan Sigrist for generously providing the GluRIIA[E783A] mutant flies, and the Iowa Developmental Studies Hybridoma Bank for GluRIIA antibodies. This work was partially supported by an NIH/NINDS grant R01NS045628 to D.F.
Open Access This article is distributed under the terms of the Creative Commons Attribution Noncommercial License which permits any noncommercial use, distribution, and reproduction in any medium, provided the original author(s) and source are credited.

\section{References}

Augustin H, Grosjean Y, Chen K, Sheng Q, Featherstone DE (2007) Nonvesicular release of glutamate by glial xCT transporters suppresses glutamate receptor clustering in vivo. J Neurosci 27:111-123

Ball R, Xing B, Bonner P, Shearer J, Cooper RL (2003) Long-term in vitro maintenance of neuromuscular junction activity of Drosophila larvae. Comp Biochem Physiol A Mol Integr Physiol 134:247-255

Banerjee S, Pillai AM, Paik R, Li J, Bhat MA (2006) Axonal ensheathment and septate junction formation in the peripheral nervous system of Drosophila. J Neurosci 26:3319-3329

Besson MT, Soustelle L, Birman S (1999) Identification and structural characterization of two genes encoding glutamate transporter homologues differently expressed in the nervous system of Drosophila melanogaster. FEBS Lett 443:97-104

Besson MT, Soustelle L, Birman S (2000) Selective high-affinity transport of aspartate by a Drosophila homologue of the excitatory amino-acid transporters. Curr Biol 10:207-210

Bogdanik L, Mohrmann R, Ramaekers A, Bockaert J, Grau Y, Broadie K, Parmentier ML (2004) The Drosophila metabotropic glutamate receptor DmGluRA regulates activity-dependent synaptic facilitation and fine synaptic morphology. J Neurosci 24:91059116

Cebada J, Alvarado-Alvarez R, Becerra E, Neri-Bazan L, Rocha L, Garcia U (2006) An improved method for long-term measuring of hemolymph fluctuations of non-essential amino acids, GABA and histamine from freely moving crayfish. J Neurosci Methods 153:1-7

Chen K, Merino C, Sigrist SJ, Featherstone DE (2005) The 4.1 protein coracle mediates subunit-selective anchoring of Drosophila glutamate receptors to the postsynaptic actin cytoskeleton. J Neurosci 25:6667-6675

Clements AN, May TE (1974) Studies on locust neuromuscular physiology in relation to glutamic acid. J Exp Biol 60:673-705

Echalier G (1997) Composition of the body fluid of Drosophila and the design of culture media for Drosophila cells. Drosophila cells in culture. Academic, New York

Evans PD (1972) The free amino acid pool of the haemocytes of Carcinus maenas (L.). J Exp Biol 56:501-507

Evans PD, Crossley AC (1974) Free amino acids in the haemocytes and plasma of the larva of Calliphora vicina. J Exp Biol 61:463-472

Faeder IR, Salpeter MM (1970) Glutamate uptake by a stimulated insect nerve muscle preparation. J Cell Biol 46:300-307

Featherstone DE, Shippy SA (2008) Regulation of synaptic transmission by ambient extracellular glutamate. Neuroscientist 14:171-181

Featherstone DE, Rushton E, Broadie K (2002) Developmental regulation of glutamate receptor field size by nonvesicular glutamate release. Nat Neurosci 5:141-146

Featherstone DE, Rushton E, Rohrbough J, Liebl F, Karr J, Sheng Q, Rodesch CK, Broadie K (2005) An essential Drosophila glutamate receptor subunit that functions in both central neuropil and neuromuscular junction. J Neurosci 25:3199-3208

Grosjean Y, Grillet M, Augustin H, Ferveur JF, Featherstone DE (2008) A glial amino-acid transporter controls synapse strength and courtship in Drosophila. Nat Neurosci 11:54-61 
Heckmann M, Dudel J (1997) Desensitization and resensitization kinetics of glutamate receptor channels from Drosophila larval muscle. Biophys J 72:2160-2169

Heckmann M, Parzefall F, Dudel J (1996) Activation kinetics of glutamate receptor channels from wild-type Drosophila muscle. Pflugers Arch 432:1023-1029

Jan LY, Jan YN (1976) L-glutamate as an excitatory transmitter at the Drosophila larval neuromuscular junction. J Physiol (Lond) 262:215-236

Liebl FL, Chen K, Karr J, Sheng Q, Featherstone DE (2005) Increased synaptic microtubules and altered synapse development in Drosophila sec8 mutants. BMC Biol 3:27

Liebl FL, Werner KM, Sheng Q, Karr JE, McCabe BD, Featherstone DE (2006) Genome-wide P-element screen for Drosophila synaptogenesis mutants. J Neurobiol 66:332-347

Marrus SB, Portman SL, Allen MJ, Moffat KG, DiAntonio A (2004) Differential localization of glutamate receptor subunits at the Drosophila neuromuscular junction. J Neurosci 24:1406-1415

McDonald TJ (1975) Neuromuscular pharmacology of insects. Annu Rev Entomol 20:151-166

Petersen SA, Fetter RD, Noordermeer JN, Goodman CS, DiAntonio A (1997) Genetic analysis of glutamate receptors in Drosophila reveals a retrograde signal regulating presynaptic transmitter release. Neuron 19:1237-1248

Pierce VA, Mueller LD, Gibbs AG (1999) Osmoregulation in Drosophila melanogaster selected for urea tolerance. J Exp Biol 202:2349-2358
Piyankarage SC, Augustin H, Grosjean Y, Featherstone DE, Shippy SA (2008) Hemolymph amino acid analysis of individual Drosophila larvae. Anal Chem 80:1201-1207

Qin G, Schwarz T, Kittel RJ, Schmid A, Rasse TM, Kappei D, Ponimaskin E, Heckmann M, Sigrist SJ (2005) Four different subunits are essential for expressing the synaptic glutamate receptor at neuromuscular junctions of Drosophila. J Neurosci 25:3209-3218

Ribeiro C, Brehelin M (2006) Insect haemocytes: what type of cell is that? J Insect Physiol 52:417-429

Rival T, Soustelle L, Cattaert D, Strambi C, Iche M, Birman S (2006) Physiological requirement for the glutamate transporter dEAAT1 at the adult Drosophila neuromuscular junction. J Neurobiol 66:1061-1074

Schmid A, Qin G, Wichmann C, Kittel RJ, Mertel S, Fouquet W, Schmidt M, Heckmann M, Sigrist SJ (2006) Non-NMDA-type glutamate receptors are essential for maturation but not for initial assembly of synapses at Drosophila neuromuscular junctions. J Neurosci 26:11267-11277

Schmid A, Hallermann S, Kittel RJ, Khorramshahi O, Frolich AM, Quentin C, Rasse TM, Mertel S, Heckmann M, Sigrist SJ (2008) Activity-dependent site-specific changes of glutamate receptor composition in vivo. Nat Neurosci 11:659-666

Seal RP, Daniels GM, Wolfgang WJ, Forte MA, Amara SG (1998) Identification and characterization of a cDNA encoding a neuronal glutamate transporter from Drosophila melanogaster. Receptors Channels 6:51-64 